\title{
Randomised trial of nasal surgery for fixed nasal obstruction in obstructive sleep apnoea
}

\author{
I. Koutsourelakis*, G. Georgoulopoulos\#, E. Perraki*, E. Vagiakis*, \\ C. Roussos* and S.G. Zakynthinos*
}

ABSTRACT: Although nasal surgery has limited efficacy in obstructive sleep apnoea (OSA) treatment, some patients experience improvement. The present study tested the hypothesis that post-surgery improvement is associated with increased nasal breathing epochs.

A total of 49 OSA patients (mean apnoea/hypopnoea index (AHI) $30.1 \pm 16.3$ events $\cdot h^{-1}$ ) with symptomatic fixed nasal obstruction due to deviated septum were randomly assigned to either septoplasty (surgery group; 27 patients) or sham surgery (placebo group; 22 patients). The breathing route was examined during overnight polysomnography.

All patients in the placebo group were nonresponders, whereas in the surgery group four (14.8\%) patients were responders and exhibited considerable increase in nasal breathing epochs (epochs containing more than three consecutive phasic nasal signals), and 23 patients were nonresponders, presenting a modest increase in nasal breathing epochs. The change in $\mathrm{AHI}$ was inversely related to the change in nasal breathing epochs, with responders exhibiting among the greatest increases in nasal breathing epochs. Baseline nasal breathing epochs were positively related to per cent change in AHI. Responders had among the lowest baseline nasal breathing epochs; a cut-off value of $62.4 \%$ of total sleep epochs best separated $(100 \%$ sensitivity, $82.6 \%$ specificity) responders/nonresponders.

In conclusion, nasal surgery rarely treats obstructive sleep apnoea effectively. Baseline nasal breathing epochs can predict the surgery outcome.

KEYWORDS: Apnoea/hypopnoea index, nasal breathing epochs, nasal surgery, obstructive sleep apnoea

n humans, the nose normally accounts for approximately half of the total respiratory resistance to airflow [1]. During sleep, nasal obstruction can provoke an increase in airflow resistance upstream in the upper airways, promoting more negative intraluminal pressure in the pharynx and predisposing to pharyngeal occlusion [2]. Therefore, experimentally induced nasal obstruction triggers the generation of obstructive apnoeas, and allergic rhinitis elicits both sleep fragmentation and obstructive sleep apnoea (OSA) [3, 4].

Despite the relationship between nasal obstruction and OSA, the therapeutic effect of improving nasal airway patency on OSA severity remains a point of conjecture [5]. In fact, administration of intranasal corticosteroids has been shown to improve sleepiness and reduce the apnoea/ hypopnoea index (AHI) in patients with OSA and rhinitis [6], whereas several uncontrolled trials examining the impact of surgical correction of deviated nasal septum on OSA severity provided inconsistent and rather disappointing results [7]. The reasons for this limited efficacy of the surgical correction of nasal obstruction are unclear [8]. Interestingly, within these trials, there were OSA patients who experienced polysomnographic and symptomatic resolution following nasal surgery $[7,9]$.

The importance of the breathing route (oral or nasal) in upper airway obstruction during sleep has been well documented. Indeed, the present authors demonstrated a potent correlation between oral/oronasal breathing epochs and the number of apnoeas and hypopnoeas [10], and forced mouth breathing has been shown to exert a profound influence on OSA severity [5]. Accordingly, the change in breathing route induced by increased nasal resistance could by itself contribute to the increase in the frequency

\section{AFFILIATIONS}

*Dept of Critical Care and Pulmonary Services, Centre of Sleep Disorders, Medical School of Athens University, and

\#Ear, Nose and Throat Dept, Evangelismos Hospital, Athens, Greece.

CORRESPONDENCE

S.G. Zakynthinos

Dept of Critical Care and Pulmonary Services

Medical School of Athens University

Evangelismos Hospital

45-47 Ipsilandou Str

GR 10675

Athens

Greece

Fax: 302107216503

E-mail: szakynthinos@yahoo.com

Received:

July 122007

Accepted:

September 042007

SUPPORT STATEMENT

The present work was funded by the Thorax Foundation (Athens, Greece).

STATEMENT OF INTEREST

None declared. 
of sleep-related breathing disorders [11, 12]. Consequently, the surgical reversal of nasal obstruction and prevention of the ensuing shift to oral breathing has prompted FITZPATRICK et al. [5] to make the plausible speculation that the breathing route before and after nasal surgery might be a determinant of the surgical outcome [13].

Therefore, the purpose of the present study was to examine the effectiveness of surgical correction of nasal obstruction on alleviating OSA through a randomised placebo-controlled trial (sham surgery) and to investigate whether the breathing route before and after surgery is associated with the outcome of nasal surgery. The present authors' hypothesis was that patients with OSA who respond to nasal surgery by reducing the number of apnoeas/hypopnoeas might exhibit a preoperatively decreased proportion of nasal breathing epochs and thus greater potential for increasing them post-operatively, in comparison with patients with OSA who do not benefit from nasal surgery.

\section{METHODS}

\section{Study subjects}

A total of 51 consecutive subjects who referred to the Centre of Sleep Disorders of the "Evangelismos" General Hospital of Athens, Greece for suspected sleep-disordered breathing were recruited. Enrolment criteria were: 1) nasal septum deviation with or without inferior turbinal hypertrophy, as assessed by clinical examination and flexible fibreoptic nasopharyngoscopy along with nasal resistance values exceeding normal limits at baseline (symptomatic fixed nasal obstruction); 2) AHI $>5$ events $\cdot h^{-1}$ at baseline; 3) no upper or lower respiratory tract disease, including a history of nasal allergy; 4) no recent surgery involving the upper airways; 5) no use of medications known to influence nasal resistance (antihistamine, decongestants, etc.); and 6) no history of neuromuscular or cardiovascular disease. Exclusion criterion was the treatment of OSA with continuous positive airway pressure (CPAP) during the course of the study.

Using a table of random numbers, subjects were randomised to either the surgery group or the placebo group (sham surgery). The subjects of the placebo group were offered nasal surgery at the end of the study. One otolaryngologist performed all operations under topical anaesthesia. Due to long waiting lists for both diagnosis and treatment with CPAP (3-4 months in the latter case in the present authors' hospital), CPAP treatment in all patients included in the study, where needed, was delayed only for a period similar to that in which CPAP treatment would normally have been provided.

Prior to enrolment in the study, each participant provided informed consent, which included writing in a chart, "On entering this study, I realise that I may receive placebo surgery. I further realise that this means that I will not have nasal surgery. This placebo nasal surgery will not benefit OSA". The study protocol was approved by the hospital ethics committee.

\section{Study protocol}

Sleep studies were performed $\leqslant 1$ month before (baseline study) and 3-4 months after surgery. Each subject reported to the sleep laboratory at 21:00-22:00 h. Nasal resistance was measured in upright-seated and supine positions. A full-night diagnostic polysomnography with concomitant monitoring of the breathing route during sleep was then performed, usually 00:00-07:00 h.

\section{Rhinomanometry}

For each subject, nasal resistance to airflow was measured by active anterior rhinomanometry (PDD-301/r; Piston, Budapest, Hungary) during wakefulness, first in the upright-seated position and then in supine position using a standard protocol [10].

\section{Polysomnographic methods}

A full-night diagnostic polysomnography (EMBLA S7000; Medcare Flaga, Reykjavik, Iceland) was performed in each subject. In order to determine the stages of sleep, an electroencephalogram (with four channels: C4-A1, C3-A2, O2-A1 and O1-A2), electro-oculogram and electromyogram of the submentalis muscle were obtained. Arterial blood oxyhaemoglobin was recorded with the use of a finger pulse oximeter. Thoracoabdominal excursions were measured qualitatively by respiratory effort sensors (XactTrace ${ }^{\mathrm{TM}}$ belts featuring respiratory inductive plethysmography; Medcare Flaga) placed over the rib cage and abdomen. Snoring was detected with a vibration snore sensor, and body posture with a body position sensor. Airflow was monitored using an oral thermistor (oral flow sensor; Medcare Flaga) placed in front of the mouth and a nasal cannula/pressure transducer $(53 \mathrm{~cm}$; Medcare Flaga) inserted in the opening of the nostrils and linked to independent channels, as previously described elsewhere [10]. All variables were recorded with a digital acquisition system (Somnologica 3.3; Medcare Flaga).

\section{Surgery group}

All patients underwent submucous resection of the deviated nasal septum. In 18 out of 27 patients, submucous resection of the bilateral inferior turbinates was also performed. Nasal packing was removed on the second post-operative day, and routine saline nasal irrigation and debridement were performed. Post-operatively, none of the patients experienced any complication.

\section{Placebo group (sham surgery)}

To ensure blinding, a standard submucosal resection of the nasal septum was simulated. After the infiltration of the nasal septum with $10 \mathrm{~mL}$ lidocaine $1 \%$ containing epinephrine $1: 200,000$, the surgeon asked for all instruments and manipulated the nose as if submucosal resection was being performed. Patients remained in the operating room for the same amount of time required for the surgery group. Patients spent the night after the procedure in the hospital and were cared for by nurses who were unaware of the treatment group assignment. Nasal packing was removed on the second post-operative day and routine saline nasal irrigation and debridement were performed.

\section{End-points and definition of treatment response}

The primary end-point was the reduction in AHI in the surgery group versus the control group. In this context, treatment success was defined as a post-operative $\mathrm{AHI}$ $<15$ events $\cdot \mathrm{h}^{-1}$ along with $\geqslant 50 \%$ decrease from the baseline AHI (responders) [14]. Treatment failure was defined as a postoperative AHI $>15$ events $\cdot \mathrm{h}^{-1}$ and/or a decrease of AHI from 


\begin{tabular}{|c|c|c|c|c|}
\hline \multirow[t]{3}{*}{ TABLE 1} & values and & inographic p & at baselin & fter surgery in the \\
\hline & \multicolumn{2}{|c|}{ Surgery group } & \multicolumn{2}{|c|}{ Placebo group } \\
\hline & Baseline & After surgery & Baseline & After sham surgery \\
\hline Subjects n & 27 & & 22 & \\
\hline Body mass index $\mathrm{kg} \cdot \mathrm{m}^{-2}$ & $30.4 \pm 3.2$ & $31.0 \pm 3.6$ & $29.9 \pm 3.5$ & $30.3 \pm 3.5$ \\
\hline Apnoea/hypopnoea index events $\cdot h^{-1}$ & $31.5 \pm 16.7$ & $31.5 \pm 18.2$ & $30.6 \pm 13.8$ & $32.1 \pm 14.3$ \\
\hline \multicolumn{5}{|l|}{ Nasal resistance $\mathrm{cmH}_{2} \mathrm{O} \cdot \mathrm{L}^{-1} \cdot \mathrm{S}$} \\
\hline Seated & $4.2 \pm 0.9$ & $2.0 \pm 0.6^{\star \star}$ & $4.0 \pm 0.8$ & $4.0 \pm 0.7$ \\
\hline Supine & $4.5 \pm 1.0$ & $2.4 \pm 0.5^{\star \star}$ & $4.4 \pm 0.8$ & $4.4 \pm 0.7$ \\
\hline Nasal breathing epochs \% TSE & $71.4 \pm 13.2$ & $84.0 \pm 6.0^{* *}$ & $74.1 \pm 11.8$ & $74.3 \pm 10.6$ \\
\hline Oral breathing epochs \% TSE & $3.5 \pm 3.8$ & $1.3 \pm 1.3^{\star}$ & $3.6 \pm 2.7$ & $3.3 \pm 2.3$ \\
\hline Oronasal breathing epochs $\%$ TSE & $25.1 \pm 10.0$ & $14.8 \pm 5.4^{\star \star}$ & $22.3 \pm 9.2$ & $22.4 \pm 8.4$ \\
\hline
\end{tabular}

Data are presented as mean $\pm \mathrm{SD}$, unless otherwise stated. TSE: total sleep epochs. *: $p<0.05$ versus baseline; **: $p<0.01$ versus baseline.

baseline $<50 \%$ (nonresponders). A complete response was defined as a reduction in AHI to $\leqslant 5$ events $\cdot \mathrm{h}^{-1}$ (normalisation of AHI). The secondary end-point was daytime sleepiness, as assessed by the Epworth Sleepiness Scale (ESS) score.

\section{Analysis}

Sleep stage was scored manually in 30-s epochs [15]. Obstructive respiratory events were scored using standard criteria [16, 17]. Route of breathing was evaluated by using the oral and nasal sensor signals to classify each 30-s epoch as nasal, oral or oronasal based on the predominant breathing route, and was expressed as the percentage of total sleep epochs (TSE), as described previously [10]. Cross-contamination between the oral and nasal channel was meticulously excluded by regular testing during polysomnographic calibration. Therefore, subjects were asked to breathe normally and exclusively through the nose for $30 \mathrm{~s}$ and, subsequently, through the mouth for another $30 \mathrm{~s}$ in both supine and right lateral postures so that it could be verified that each sensor was activated exclusively. The sensors were continuously checked during the recording to avoid dislodgement. All measurements were analysed by a single investigator to ensure consistency and all polysomnographies were scored by a single experienced sleep technologist and subsequently reviewed by the same investigator, who was blinded to the patient's group identity.

The minimum sample size was calculated based on $80 \%$ power and a two-sided 0.05 significance level. Sample size capable of detecting a change of 10 events $\cdot h^{-1}$ for AHI after surgery was estimated using a mean $\pm \mathrm{SD}$ baseline $\mathrm{AHI}$ value of $36 \pm 14$ events $\cdot h^{-1}$, which was obtained from a previous study [18]. The critical sample size was estimated to be 24 patients. Data are presented as mean $\pm \mathrm{SD}$, unless otherwise specified. Baseline difference between groups concerning age was tested by unpaired t-test. Two-way ANOVA with repeated measures was used for variable comparisons between groups before and after surgery, followed by the Scheffé test for post hoc analyses, as appropriate. Linear regression analysis was performed using the least squares method. Diagnostic performance of baseline nasal breathing epochs to distinguish responders from nonresponders to surgery was expressed as the area under the receiver operating characteristic curve. A value of 1.0 indicates perfect discrimination. Cut-off value achieving the best combination of sensitivity and specificity was calculated as the maximum difference between sensitivity and 1-specificity. A p-value $<0.05$ was considered statistically significant.

\section{RESULTS}

Of the 51 patients (39 males) initially fulfilling the inclusion criteria, 49 were considered eligible for further analysis. Two patients dropped out due to willingness to begin CPAP therapy. Of the 49 patients, 27 were randomly assigned to the surgery group and 22 to the placebo group. Baseline characteristics were similar in the two study groups (table 1). There was no change in body mass index after surgery or sham surgery. Nasal resistance decreased in both seated and supine positions in the surgery group $(\mathrm{p}<0.001)$, whereas in the placebo group it remained unchanged.

\section{Apnoea/hypopnoea index}

AHI remained unchanged after surgery or sham surgery (table 1). Based on the pre-study end-points and definitions of treatment response, $23(85.2 \%)$ patients of the surgery group were treatment failures (nonresponders), whereas four $(14.8 \%)$ patients were treatment successes (responders; table 2 and fig. 1). Only one $(3.7 \%)$ patient of the surgery group showed complete response. AHI decreased in responders and increased in nonresponders $(p<0.001$, two-way ANOVA; table 2$)$. All patients of the placebo group were treatment failures (fig. 1). 
TABLE 2 Anthropometric data, nasal resistance values and polysomnographic parameters at baseline and after surgery in the responders and nonresponders

\begin{tabular}{|c|c|c|c|c|}
\hline & \multicolumn{2}{|c|}{ Responders } & \multicolumn{2}{|c|}{ Nonresponders } \\
\hline & Baseline & After surgery & Baseline & After surgery \\
\hline Male sex \% & 75.0 & & 60.9 & \\
\hline Age yrs & $36.3 \pm 5.9$ & & $39.4 \pm 7.8$ & \\
\hline Body mass index $\mathrm{kg} \cdot \mathrm{m}^{-2}$ & $27.8 \pm 4.3$ & $28.0 \pm 4.3$ & $30.9 \pm 2.9^{\#}$ & $31.5 \pm 3.3$ \\
\hline \multicolumn{5}{|l|}{ Nasal resistance $\mathrm{cmH}_{2} \mathrm{O} \cdot \mathrm{L}^{-1} \cdot \mathrm{S}$} \\
\hline Seated & $4.3 \pm 0.7$ & $1.8 \pm 0.3^{\star}$ & $4.2 \pm 0.9$ & $2.1 \pm 0.6^{*}$ \\
\hline Supine & $4.8 \pm 0.7$ & $2.3 \pm 0.2^{\star}$ & $4.5 \pm 1.0$ & $2.4 \pm 0.6^{*}$ \\
\hline Average oxygen saturation $\%$ & $95.1 \pm 1.0$ & $96.1 \pm 0.9$ & $94.2 \pm 1.0$ & $94.2 \pm 1.2$ \\
\hline Duration of apnoea/hypopnoea s & $22.3 \pm 2.5$ & $23.1 \pm 3.6$ & $23.4 \pm 3.5$ & $23.6 \pm 3.2$ \\
\hline Epworth Sleepiness Scale score & $16.0 \pm 3.6$ & $11.8 \pm 5.0^{*}$ & $13.0 \pm 2.6^{\#}$ & $11.7 \pm 3.2$ \\
\hline
\end{tabular}

Data are presented as mean $\pm S D$, unless otherwise stated. TSE: total sleep epochs. *: $p<0.05$ versus baseline; ${ }^{*}: p<0.05$ versus baseline of responders; ${ }^{* * *}: p<0.001$ versus baseline of responders.

\section{ESS score}

ESS score was the same in patients of the surgery and placebo groups at baseline and decreased after treatment only in the surgery group (table 1). Baseline ESS score was lower in nonresponders than in responders, and decreased after nasal surgery only in responders $(\mathrm{p}<0.001$; table 2$)$. Individual values of ESS score, along with nasal resistance and AHI of patients in the surgery group, are contained in table 3.

\section{Nasal and oral/oronasal breathing epochs}

Baseline proportion of nasal and oral/oronasal breathing epochs did not differ in patients of the surgery and placebo

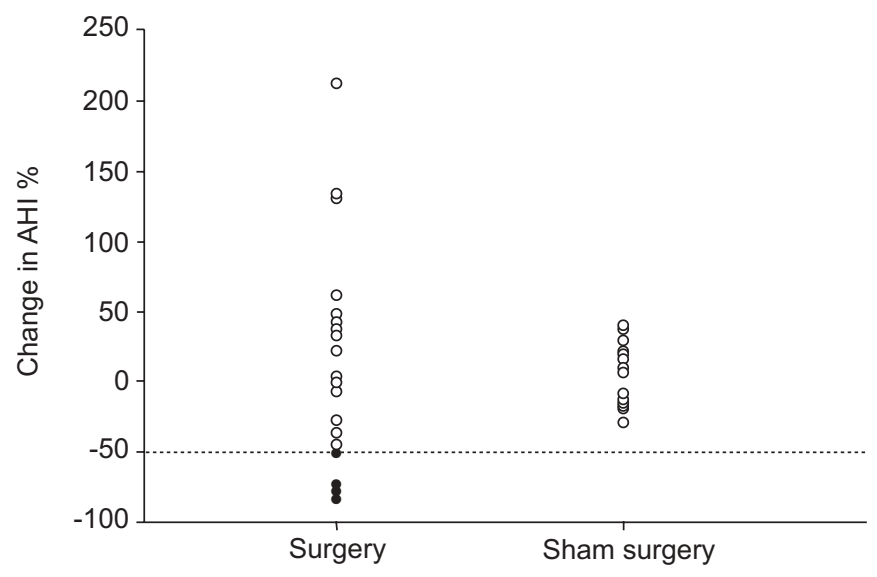

FIGURE 1. Change of apnoea/hypopnoea index (AHI) in patients of the surgery group and the placebo group (sham surgery). - responders; $\mathrm{O}$ : nonresponders; $\cdots \cdots \cdot$. limit of per cent change in $\mathrm{AHI}$ after surgery compared with baseline $(-50 \%)$. This limit, along with a post-operative $A H I<15$ events $\cdot h^{-1}$, was used to define treatment response. groups but, after surgery, nasal breathing epochs increased and oral/oronasal breathing epochs decreased in the surgery group, whereas they remained unchanged in the placebo group $(\mathrm{p}<0.05$; table 1$)$. Baseline oral/oronasal breathing epochs were lower and nasal breathing epochs were higher in nonresponders compared with the respective baseline values in responders (table 2). Most importantly, baseline nasal breathing epochs could discriminate responders from nonresponders; the mean $\pm \mathrm{SD}$ area under the receiver operating characteristic curve was $0.924 \pm 0.054$, and the cut-off value of nasal breathing epochs at baseline that best separated $(100 \%$ sensitivity and $82.6 \%$ specificity) responders from nonresponders was $62.4 \%$ of TSE (fig. 2). Individual values of nasal breathing epochs at baseline and after surgery in responders and nonresponders are also illustrated in figure 2. Nasal breathing epochs increased, whereas oral/oronasal breathing epochs decreased in both groups, but they changed in a significantly greater degree in responders than in nonresponders $(p<0.001$; fig. 3$)$. The change in nasal breathing epochs after surgery was inversely related to the change in AHI $\left(r^{2}=0.775, p<0.001\right.$; fig. 4); responders exhibited the greatest increases in nasal breathing epochs after surgery. Finally, baseline nasal breathing epochs were positively related to the per cent change in AHI $\left(\mathrm{r}^{2}=0.610, \mathrm{p}<0.001\right.$; fig. 5).

\section{DISCUSSION}

The main findings of the present randomised controlled trial of surgical correction of fixed nasal obstruction in OSA were as follows. 1) Nasal surgery does not influence AHI, since only $15 \%$ of patients experienced significant reduction in $\mathrm{AHI}$ (responders) and only $4 \%$ of patients had a complete response (normalisation of AHI). 2) The change in AHI after surgery was inversely related to the change in nasal breathing epochs, so that the increase in nasal breathing epochs explained $77.5 \%$ 


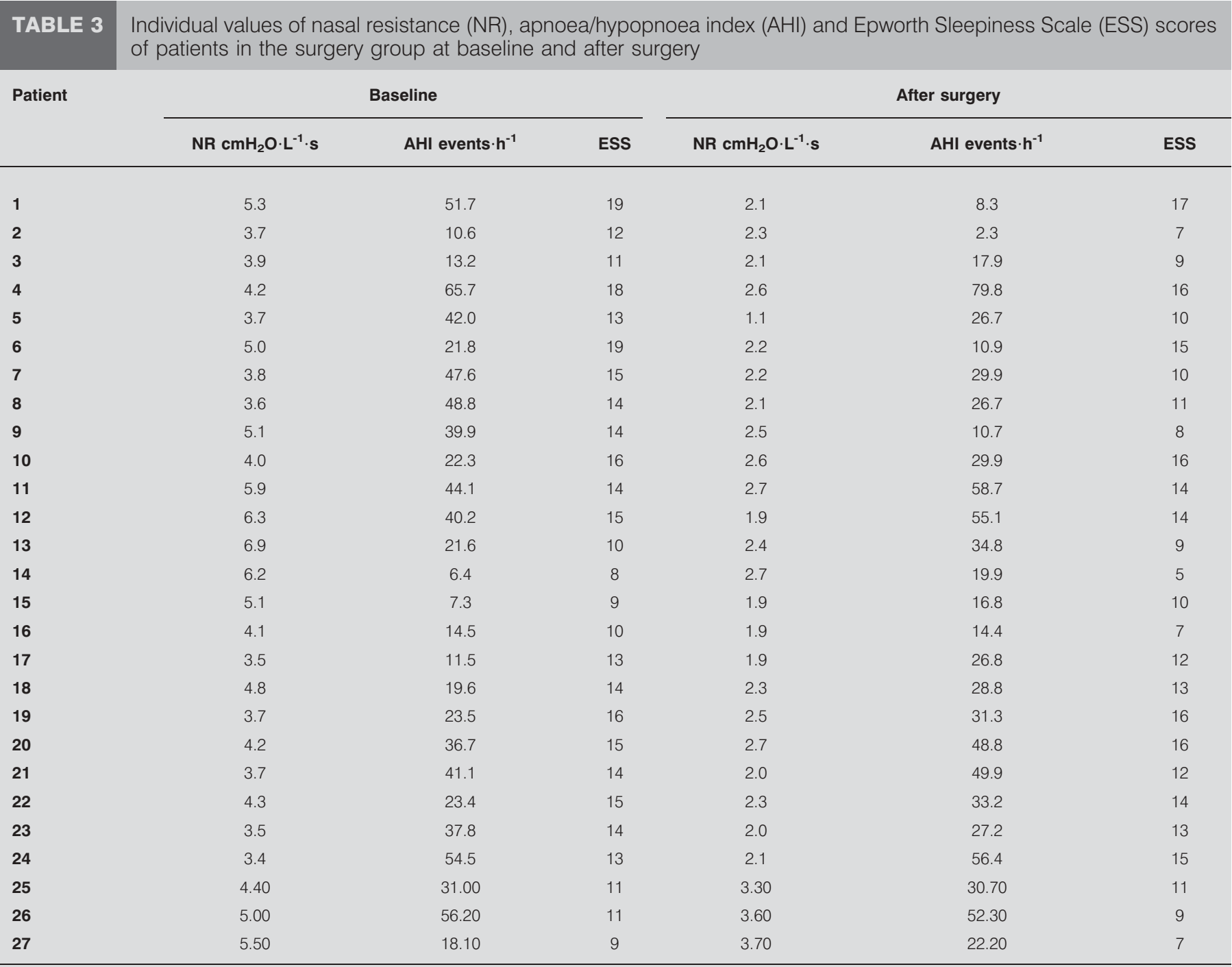

Patients 1, 2, 6 and 9 fulfilled the criteria for treatment success (responders).

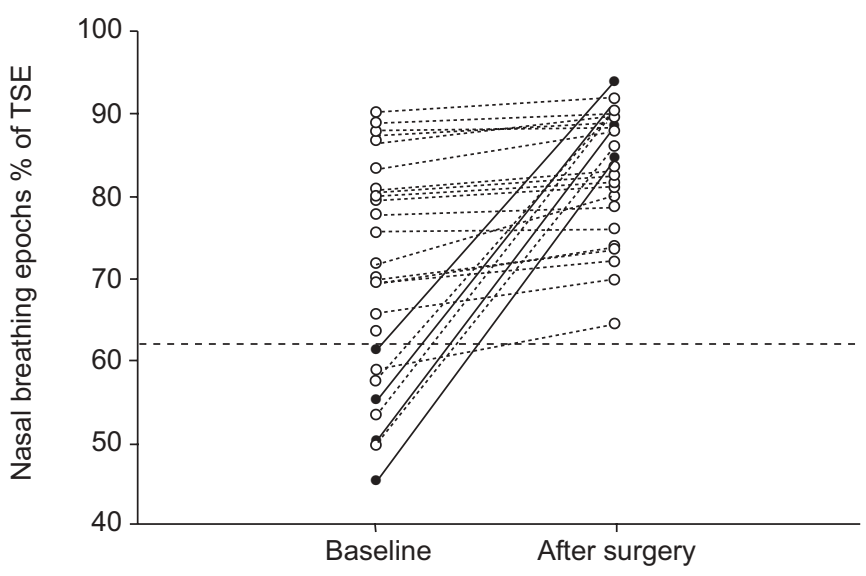

FIGURE 2. Nasal breathing epochs at baseline and after surgery in responders $(\bullet)$ and in nonresponders $(\bigcirc)$. - - -: cut-off value of baseline nasal breathing epochs $(62.4 \%$ of total sleep epochs (TSE)) with maximum diagnostic discrimination of response to surgery (100\% sensitivity and $82.6 \%$ specificity). of variance of the decrease in AHI. Responders exhibited among the greatest increases in nasal breathing epochs after surgery. 3) Baseline nasal breathing epochs were positively related to the per cent change in AHI after surgery, so that lower baseline nasal breathing epochs explained $61.0 \%$ of variance of the per cent decrease in AHI after surgery. Responders had among the lowest baseline nasal breathing epochs. 4) Baseline nasal breathing epochs with a cut-off value of $62.4 \%$ of TSE separated responders from nonresponders with $100 \%$ sensitivity and $82.6 \%$ specificity.

The present study is the first to examine the role and prove the rare efficacy of nasal surgery in the treatment of OSA in a randomised controlled setting with sham surgery. In fact, in the absence of a control group, any result obtained by previous trials may have been attributed not only to the intrinsic effect of the surgical procedure but also to either the natural history of the condition or an independent placebo effect [19]. In general, previous studies were prospective pre- and postsurgery comparisons $[7,9,18,20,21]$ or retrospective case series $[22,23]$, where the surgical procedures employed were 

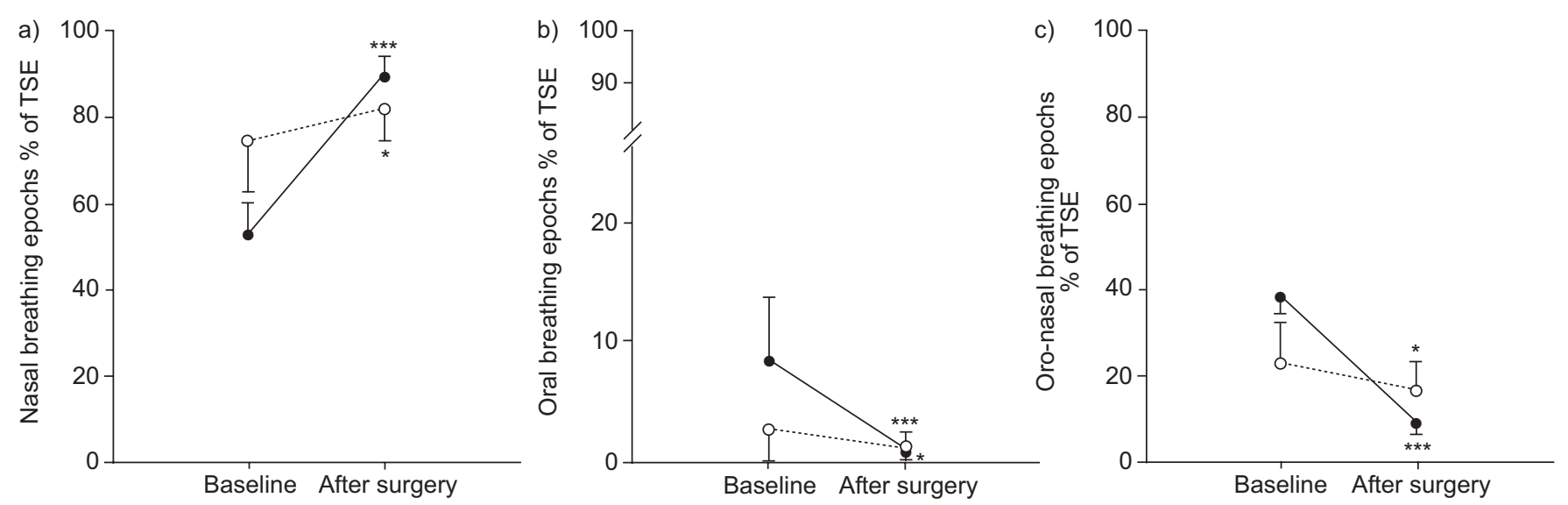

FIGURE 3. Occurrence of a) nasal, b) oral and c) oronasal breathing epochs at baseline and after surgery. Data are presented as mean \pm SD. $O$ : nonresponders. TSE: total sleep epochs. ${ }^{*}: p<0.05$ versus baseline; ${ }^{* *}: p<0.001$ versus baseline.

heterogeneous $[7,20,21]$ and objective assessment of nasal resistance was often not performed in all patients [18, 21, 22]. In particular, in an analysis of nine previous studies, $18 \%$ of patients achieved a response defined by a post-operative AHI $<20$ events $\cdot h^{-1}$ along with $\geqslant 50 \%$ decrease from the baseline AHI [7], a finding modestly better than that of the present study $(15 \%$ of the current patients fulfilled this definition of response). Taking into account the combination of these results with the findings of the present study, it appears that septoplasty, with or without bilateral inferior turbinate resection, has indeed a limited role in the management of sleep-disordered breathing. However, the role of nasal surgery in facilitating the initiation of CPAP therapy is unequivocal [20], since it has been proven that the CPAP needed to reverse OSA post-operatively is significantly reduced [21].

In respect to the role of nasal breathing epochs, the present study can have important ramifications for clinical practice, since nasal surgery has a significantly variable effect on OSA

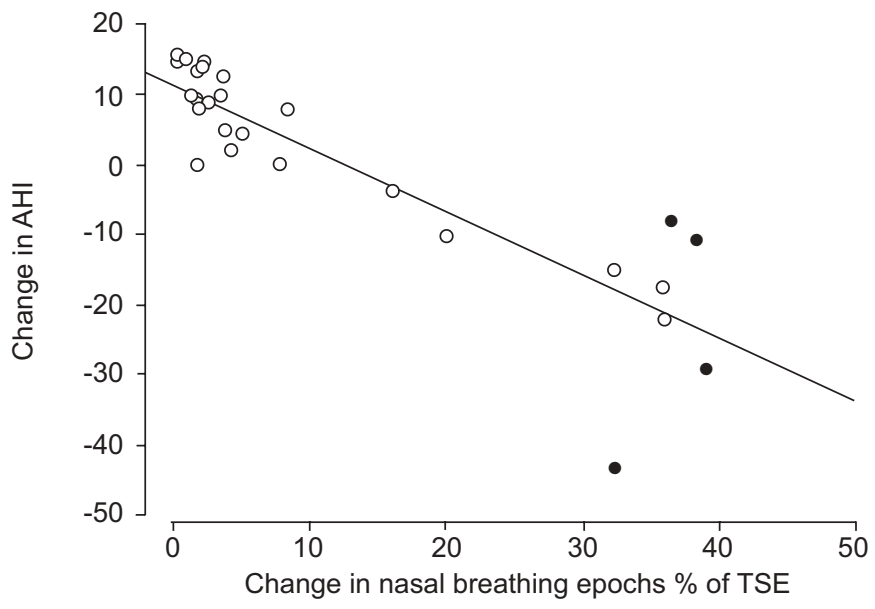

FIGURE 4. Relationship between the change in nasal breathing epochs and the change in apnoea/hypopnoea index (AHI) before and after surgery in responders $(\bullet)$ and in nonresponders $(\bigcirc)$. — : linear regression line $\left(r^{2}=0.775 ; p<0.001\right)$. TSE: total sleep epochs. ranging from complete resolution to severe aggravation. Therefore, the ability of baseline nasal breathing epochs to predict therapeutic response is of paramount importance. This inconsistent effect of nasal surgery on OSA is not novel [7], but is in line with the assumption that pharyngeal collapsibility is sensitive to alterations in upper airway anatomy through both local mechanical factors and neuromuscular tone changes [24, 25]. Hence, it appears that nasal surgery should be avoided in OSA patients with nasal obstruction when baseline proportion of nasal breathing epochs is more than $\sim 62 \%$ of TSE (fig. 2). In this context, although the association between nasal obstruction and the proportion of nasal breathing is intuitively obvious [5], a moderate increase in nasal resistance may not induce a decrease in nasal breathing but a "through high nasal resistance" breathing pattern [13]. Similarly, OHKI et al. [26] concluded that in normal subjects a very high resistive load needs to be added to the nasal breathing circuit to provoke a shift to pure mouth breathing. These observations give credence to the contention that nasal resistance is only important to the point where it exceeds a certain threshold and triggers the shift to oronasal breathing. Therefore, as nasal resistance in the present study was equal in responders and nonresponders at baseline, it is plausible to suggest that responders had lower threshold than nonresponders, thereby presenting decreased proportion of nasal breathing epochs for a given value of nasal resistance. Moreover, the increase in nasal breathing epochs post-operatively might also be a surrogate for reduced mouth opening. Indeed, the latter has been convincingly associated in previous studies with sleep disordered breathing through many causal pathways [10].

It is noteworthy that several lines of evidence support the argument that mouth breathing induced by nasal obstruction facilitates the induction of apnoeas $[5,10,11]$. FITZPATRICK and co-workers $[5,11]$ documented a marked increase in OSA severity in mouth breathing as compared with nasal breathing, the normal pathway for ventilation during sleep. Furthermore, the proportion of oral and oronasal breathing epochs has been shown to be positively related to the severity of sleep disordered breathing [10]. The results of the current study add weight to the same argument, demonstrating that surgical reversal of nasal obstruction improves OSA only when it 


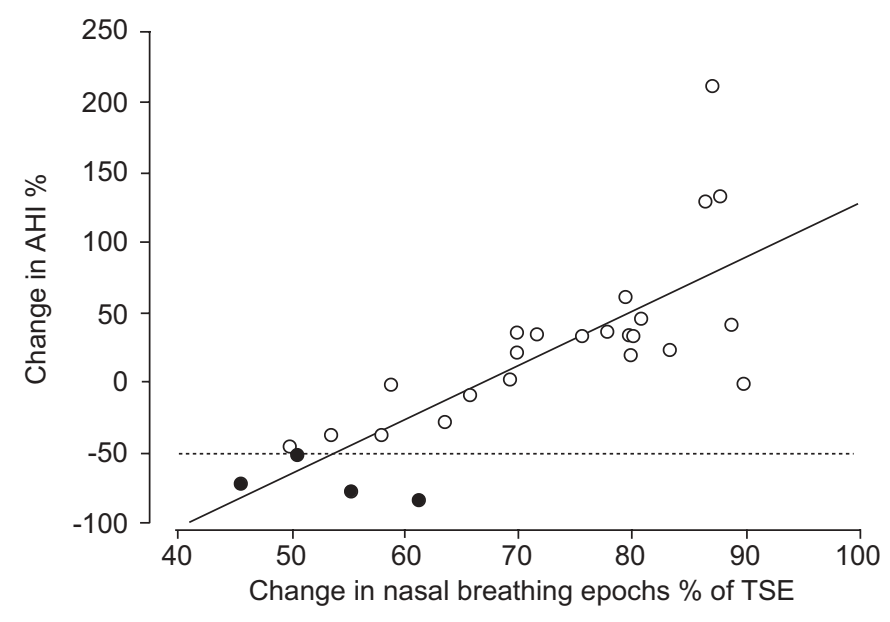

FIGURE 5. Relationship between the baseline (before surgery) nasal breathing epochs and the per cent change in apnoea/hypopnoea index (AHI) before and after surgery in responders $(\bullet)$ and in nonresponders $(\bigcirc)$. _ $\left(r^{2}=0.610 ; p<0.001\right) ; \cdots \cdots \cdot$ : limit of per cent change in $\mathrm{AHI}$ after surgery compared with baseline (-50\%). This limit, along with a post-operative $\mathrm{AHI}<15$ events $\cdot \mathrm{h}^{-1}$, was used to define treatment response.

succeeds in restoring the preponderance of nasal breathing epochs post-operatively.

Patients who underwent nasal surgery, in contrast to those who underwent sham surgery, experienced a significant reduction in the level of daytime sleepiness (table 1). However, this improvement was confined to responders, whereas in nonresponders the ESS score remained unchanged (table 2). The improvement observed in daytime somnolence may be related not only to the improvement in sleep apnoea severity but also to a relief of nasal discomfort associated with nasal obstruction. Indeed, CRAIG et al. [27] documented that reducing nasal congestion with topical corticosteroids in patients with allergic rhinitis improved subjective sleep quality.

Some methodological issues require consideration in the current study. Foremost, it is a short-term study with a follow-up period ranging $3-4$ months. It is possible that the longer-term effect of relieving nasal obstruction on OSA severity might be different. Nonetheless, Verse and PIRSIG [28] reviewed the outcome of nasal surgery as a treatment for OSA in nine studies where the follow-up period varied 144 months and failed to discern any trend towards OSA severity improvement with longer follow-up. Moreover, the study was adequately powered to detect an improvement in AHI of 10 events $\cdot h^{-1}$, as any less improvement is unlikely to be clinically relevant. Additionally, nasal resistance was measured in seated and supine positions using anterior rhinomanometry alone. Anterior rhinomanometry requires minimal cooperation and thus has increased reproducibility and negligible failure rate, although posterior nasal malformations cannot be determined. Nonetheless, posterior rhinomanometry was performed in all patients, but the results were not always acceptable. However, thanks to flexible fibreoptic nasopharyngoscopy, the possibility of posterior nasal malformations was convincingly eliminated and the results of posterior rhinomanometry were accordingly omitted from further analysis. Furthermore, the instrumentation of nasal cannula/ pressure transducer and oral thermistor to detect airflow presents some drawbacks that have been thoroughly discussed previously [10]. Although these devices are nonobtrusive and easily tolerated, they cannot quantify ventilation, partly because their signal-flow relationship is nonlinear, resulting in underestimation of nasal ventilation and overestimation of oral ventilation, especially at low flows $[29,30]$. Therefore, it would be possible that oral-only breathing may still have a nasal component and any detection of oral-only breathing might actually be scarce. Consequently, the frequency of oralonly breathing epochs could be overestimated in the present study, although it was already rarely encountered. Finally, although sensor dislodgement from the nares or from the mouth was meticulously checked by the technician on duty, it is possible that slight deviations in thermistor position may not have been completely avoided and this may have then resulted in nasal airflow contamination of the oral signal.

In conclusion, the present randomised controlled study provided evidence that nasal surgery is not, in general, effective in the treatment of obstructive sleep apnoea, since only $\sim 15 \%$ of patients presented a post-operative apnoea/ hypopnoea index of $<15$ events $\cdot h^{-1}$, along with $\geqslant 50 \%$ decrease from baseline apnoea/hypopnoea index, and only $4 \%$ of patients presented a complete polysomnographic resolution. However, the proportion of nasal breathing epochs pre-operatively can accurately delineate the subgroup of patients that can benefit from nasal surgery. Further studies are needed to confirm prospectively the performance of the cut-off point of baseline nasal breathing epochs detected in the present study to predict surgery outcome.

\section{REFERENCES}

1 Proctor DF. The upper airways. I. Nasal physiology and defence of the lungs. Am Rev Respir Dis 1977; 115: 97-129.

2 Ryan CM, Bradley TD. Pathogenesis of obstructive sleep apnea. J Appl Physiol 2005; 99: 2440-2450.

3 Carskadon MA, Bearpark HM, Sharkey KM, et al. Effects of menopause and nasal occlusion on breathing during sleep. Am J Respir Crit Care Med 1997; 155: 205-210.

4 McNicholas WT, Tarlo S, Cole P, et al. Obstructive apneas during sleep in patients with seasonal allergic rhinitis. Am Rev Respir Dis 1982; 126: 625-628.

5 Fitzpatrick MF, McLean H, Urton AM, Tan A, O'Donnell D, Driver HS. Effect of nasal or oral breathing route on upper airway resistance during sleep. Eur Respir J 2003; 22: 827-832.

6 Kiely JL, Nolan P, McNicholas WT. Intranasal corticosteroid therapy for obstructive sleep apnoea in patients with co-existing rhinitis. Thorax 2004; 59: 50-55.

7 Verse T, Maurer JT, Pirsig W. Effect of nasal surgery on sleep-related breathing disorders. Laryngoscope 2002; 112: 64-68.

8 Ryan CF. Sleep 9: an approach to treatment of obstructive sleep apnoea/hypopnoea syndrome including upper airway surgery. Thorax 2005; 60: 595-604.

9 Sériès F, Pierre S, Carrier G. Surgical correction of nasal obstruction in the treatment of mild sleep apnoea: 
importance of cephalometry in predicting outcome. Thorax 1993; 48: 360-363.

10 Koutsourelakis I, Vagiakis E, Roussos C, Zakynthinos S. Obstructive sleep apnoea and oral breathing in patients free of nasal obstruction. Eur Respir J 2006; 28: 1222-1228.

11 Fitzpatrick MF, Driver HS, Chatha N, Voduc N, Girard AM. Partitioning of inhaled ventilation between the nasal and oral routes during sleep in normal subjects. J Appl Physiol 2003; 94: 883-890.

12 Meurice JC, Marc I, Carrier G, Sériès F. Effects of mouth opening on upper airway collapsibility in normal sleeping subjects. Am J Respir Crit Care Med 1996; 153: 255-259.

13 McLean HA, Urton AM, Driver HS, et al. Effect of treating severe nasal obstruction on the severity of obstructive sleep apnoea. Eur Respir J 2005; 25: 521-527.

14 Sher AE, Schechtman KB, Piccirillo JF. The efficacy of surgical modifications of the upper airway in adults with obstructive sleep apnea syndrome. Sleep 1996; 19: 156-177.

15 Rechtschaffen A, Kales A, eds. A Manual of Standardized Terminology, Techniques and Scoring System for Sleep Stages of Human Subjects. Bethesda, National Institutes of Health, 1968.

16 Sleep-related breathing disorders in adults: recommendations for syndrome definition and measurement techniques in clinical research. The Report of an American Academy of Sleep Medicine Task Force. Sleep 1999; 22: 667-689.

17 Meoli AL, Casey KR, Clark RW, et al. Hypopnea in sleepdisordered breathing in adults. Sleep 2001; 24: 469-470.

18 Kim ST, Choi JH, Jeon HG, Cha HE, Kim DY, Chung YS. Polysomnographic effects of nasal surgery for snoring and obstructive sleep apnea. Acta Otolaryngol 2004; 124: 297-300.

19 Vickers AJ, de Craen AJ. Why use placebos in clinical trials? A narrative review of the methodological literature. J Clin Epidemiol 2000; 53: 157-161.

20 Sériès F, Pierre S, Carrier G. Effects of surgical correction of nasal obstruction in the treatment of obstructive sleep apnea. Am Rev Respir Dis 1992; 146: 1261-1265.
21 Friedman M, Tanyeri H, Lim J, Landsberg R, Vaidyanathan K, Caldarelli D. Effect of improved nasal breathing on obstructive sleep apnea. Otolaryngol Head Neck Surg 2000; 122: 71-74.

22 Utley DS, Shin EJ, Clerk AA, Terris DJ. A cost-effective and rational surgical approach to patients with snoring, upper airway resistance syndrome, or obstructive sleep apnea syndrome. Laryngoscope 1997; 107: 726-734.

23 Rubin AH, Eliaschar I, Joachim Z, Alroy G, Lavie P. Effects of nasal surgery and tonsillectomy on sleep apnea. Bull Eur Physiopathol Respir 1983; 19: 612-615.

24 Kirkness JP, Schwartz AR, Patil SP, et al. Dynamic modulation of upper airway function during sleep: a novel single-breath method. J Appl Physiol 2006; 101: 1489-1494.

25 Schneider H, Boudewyns A, Smith PL, et al. Modulation of upper airway collapsibility during sleep: influence of respiratory phase and flow regimen. J Appl Physiol 2002; 93: 1365-1376.

26 Ohki M, Usui N, Kanazawa H, Hara I, Kawano K. Relationship between oral breathing and nasal obstruction in patients with obstructive sleep apnea. Acta Otolaryngol Suppl 1996; 523: 228-230.

27 Craig TJ, Teets S, Lehman EB, Chinchilli VM, Zwillich C. Nasal congestion secondary to allergic rhinitis as a cause of sleep disturbance and daytime fatigue and the response to topical nasal corticosteroids. J Allergy Clin Immunol 1998; 101: 633-637.

28 Verse T, Pirsig W. Impact of impaired nasal breathing on sleep-disordered breathing. Sleep Breath 2003; 7: 63-76.

29 Farré R, Montserrat JM, Rotger M, Ballester E, Navajas D. Accuracy of thermistors and thermocouples as flowmeasuring devices for detecting hypopnoeas. Eur Respir J 1998; 11: 179-182.

30 Montserrat JM, Farré R, Ballester E, Felez MA, Pastó M, Navajas D. Evaluation of nasal prongs for estimating nasal flow. Am J Respir Crit Care Med 1997; 155: 211-215. 